\title{
Studies on surfactant function and its environmental science \# $^{\#}$
}

\author{
Hiroto TAMURA* \\ Department of Environmental Bioscience, School of Agriculture, Meijo University, Nagoya 468-8502, Japan
}

(Accepted February 14, 2011)

\begin{abstract}
The fact that the surfactant, octylphenol polyethoxylates $\left(\mathrm{OPEO}_{\mathrm{n}}\right)$, selectively solubilized epicuticular wax suggested the enhanced performance of foliar-applied agrochemicals. The biodegradation process of $\mathrm{OPEO}_{\mathrm{n}}$ by exoterminal oxidation and subsequent ether cleavage of the $\mathrm{EO}$ chain was precisely characterized by the identification of the key intermediate and the cloned alcohol oxidase. Environmental elements, such as $\mathrm{Ca}^{2+}, \mathrm{Mg}^{2+}$ and $\mathrm{Fe}^{3+}$, may influence the activity and rate of biodegradation in the environment. A highly reliable method for phylogenetic analysis at the strain level was developed using ribosomal subunit proteins, as theoretical biomarkers, encoded in the S10-spc-alpha operon with a validation procedure. (O Pesticide Science Society of Japan
\end{abstract}

Keywords: alkylphenol polyethoxylates, environmental fate, MALDI TOF MS, phylogenetic tree, ribosomal protein, endocrine disruptor.

\section{Introduction}

Chemicals contribute greatly to our life and development; however, chemicals can have adverse effects on our health and ecosystem when managed inappropriately in each process of the life cycle of the chemical i.e. manufacturing, use and disposal. Therefore, the life cycle impact assessment (LCIA) of the chemical is important not only to identify the benefits of the chemical but also to resolve potential issues in advance in order to establish a safe and sustainable society without damaging either humans or the ecosystem.

The active ingredient of agrochemicals is a good example of appropriate management by each process in the LCIA. Although a larger volume of agricultural adjuvants is commonly used in the application of agrochemicals to improve performance rather than the active ingredient itself, scientific knowledge of their function and environmental fate in the LCIA has been insufficient; therefore, they were studied to identify how adjuvants enhance the performance of agrochemicals and to elucidate their environmental science.

1. Function: evidence of surfactant solubilization of plant epicuticular wax

All aerial plant organs are covered with a nonliving, lipoidal

\footnotetext{
\# See Part II for the full Japanese article

* To whom correspondence should be addressed

E-mail: hiroto@meijo-u.ac.jp

(C) Pesticide Science Society of Japan
}

cuticular membrane that serves as the interface between the plant and its environment. Epicuticular wax (ECW) is the prime barrier against water loss from the plant and the entry of foliar-applied compounds. Although there is considerable evidence that surfactants markedly alter the fine structure of ECW, there was no direct evidence that surfactants solubilized ECW. Since ECW have particular roles (e.g., retention, spreading, distribution, and penetration) in the performance of applied agrochemicals, we investigated whether surfactants solubilized ECW in a model system. The solubilization of isolated, reconstituted tomato (Lycopersicon esculentum Mill.) fruit and broccoli (Brassica oleracaea var. botrytis L.) leaf ECWs by a nonionic surfactant, octylphenol polyethoxylates $\left(\mathrm{OPEO}_{\mathrm{n}}\right)$, was demonstrated using pyrene as a fluorescent probe. Both ECWs were solubilized at or above the surfactant critical micelle concentration. The solubilization (S) of ECWs increased with temperature $(\mathrm{T})\left(22-32^{\circ} \mathrm{C}: \mathrm{S}=1.40-0.037 \mathrm{~T}\right.$, $\left.r^{2}=0.985\right)$, and depended on their hydrophobic and lipophilic balance (HLB) $\left(\mathrm{S}=1.20-0.22 \mathrm{HLB}+0.10 \mathrm{HLB}^{2}, r^{2}=0.938\right)$. $\mathrm{OPEO}_{\mathrm{n}}$ solubilized the unsaturated hydrocarbons, hexacosene and tritriacontadiene, and the triterpenols, $\alpha$ - and $\beta$-amyrins at or above the critical micelle concentration while only traces or non-detectable quantities of saturated hydrocarbons were solubilized. Our data provided definitive evidence that $\mathrm{OPEO}_{\mathrm{n}}$ selectively solubilizes constituents of ECW. To further characterize the selective surfactant solubilization mechanism, a reconstitution model was constructed using cholesterol as the model compound of soluble ECWs, such as amyrins, and dotriacontane as a representative insoluble constituent. The mi- 
croenvironment of micelles related to the polyoxyethylene moiety has an important effect on surfactant solubilization and the octylphenol moiety must be capable of recognizing and adsorbing to a specific region of the reconstituted membrane. This selective solubilization may be related to the diverse penetration of agrochemicals.

\section{Environmental science: environmental fate of alkylphenol polyethoxylates} Although alkylphenol polyethoxylates $\left(\mathrm{APEO}_{\mathrm{n}}\right)$ have been suspected as a putative source of alkylphenol (AP) contamination, their precise environmental fate on arable land has remained unclear. Accordingly, the detailed microbial degradation of $\mathrm{APEO}_{\mathrm{n}}$ was well characterized by a pure culture of Pseudomonas putida strain S5 isolated from a Japanese paddy field under aerobic conditions, which proceeds via the successive exoscission of the ethylene oxide chain accompanied with oxidation of the hydroxyl terminal side through their carboxylic intermediates, which was confirmed using ${ }^{18} \mathrm{O}-\mathrm{la}-$ beled water and matrix-assisted laser desorption/ionization mass spectrometry (MALDI-TOF MS) analysis. The degradation process was also precisely confirmed by the cloned alcohol dehydrogenase 1 (ADH1), which has both alcohol and aldehyde dehydrogenase activity, with a flavin-binding motif and signature patterns conserved in type III FAD-dependent alcohol oxidase. The characterization of $\mathrm{OPEO}_{\mathrm{n}}$ biodegradation by $P$. putida $\mathrm{S} 5$ revealed that more carboxylic acid metabolites $\left(\mathrm{OPEC}_{2}\right)$ were accumulated in medium supplemented with only $\mathrm{Ca}^{2+}$ as compared with medium supplemented only with $\mathrm{Mg}^{2+}$, in which $\mathrm{OPEO}_{2}$ was the major metabolite. Another bacterium, Sphingomonas sp. strain BSN22, isolated from bean fields, degraded $\mathrm{OPEO}_{\mathrm{n}}$ to octylphenol (OP) under aerobic conditions. The biodegradation mechanism proceeded by the following 2 step degradation processes: step 1: degradation of $\mathrm{OPEO}_{\mathrm{n}}$ to octylphenol triethoxylate $\left(\mathrm{OPEO}_{3}\right)$, step 2: degradation from $\mathrm{OPEO}_{3}$ to OP via octylphenoxy acetic acid $\left(\mathrm{OPEC}_{1}\right)$, whose chemical structure was confirmed by mechanism-based analysis using ${ }^{18} \mathrm{O}$-labeled water. Quantitative studies revealed that $\mathrm{Mg}^{2+}$ and $\mathrm{Ca}^{2+}$ were essential for the biodegradation of $\mathrm{OPEO}_{\mathrm{n}}$. Furthermore, the rate of step 2 biodegradation was markedly accelerated by $\mathrm{Fe}^{3+}$ and the accumulated amounts of endocrine active chemicals, such as $\mathrm{OP}, \mathrm{OPEO}_{1}$ and $\mathrm{OPEC}_{1}$, significantly increased in the presence of $\mathrm{Fe}^{3+}$. Taken together, those environmental elements significantly influence the resultant ecotoxicity as well as the rate of their biodegradation in the environment because OP and $\operatorname{OPEO}_{n}(n=1,2)$ act as endocrine-active substances with both estrogen agonistic and androgen antagonistic activities. This study on the LCA of
$\mathrm{OPEO}_{\mathrm{n}}$ may play an important role in the understanding and management of the safety environment, including drinking water safety.

To understand the extent of natural adaptation to chemical exposure within a microbial consortium, therefore, we scrutinized the dynamic relationship between the microbial diversity and biodegradation capacity of $\mathrm{OPEO}_{\mathrm{n}}$, using enrichment cultures of various sediments from the Iwata River system. The results suggested that $\mathrm{OPEO}_{\mathrm{n}}$-degrading bacteria commonly exist in Iwata River, regardless of the history of spray carriers of $\mathrm{APEO}_{\mathrm{n}}$. Moreover, if excess $\mathrm{OPEO}_{\mathrm{n}}$ were applied, the degrading bacteria would be enriched and produce endocrine-active substances because of changes in microbial diversity with increasing $\mathrm{OPEO}_{\mathrm{n}}$-degrading bacteria by influencing the natural adaptation of microbial consortia. To monitor and/or regulate the environmental fate of $\mathrm{OPEO}_{\mathrm{n}}$, therefore, our study focused on developing a method of phylogenetic analysis at species and strain levels with theoretical evidence. A rapid and simple bacterial classification method by MALDI-TOF MS using ribosomal subunit proteins coded in S10-spc-alpha operon as biomarkers, because the operon encodes half of the ribosomal subunit and is highly conserved in eubacterial genomes, gave the same phylogenetic tree based on the 16S rRNA sequence. Our proposed method, named the S10-GERMS method, was performed in three steps as follows: 1) bacteria are identified by MALDI-TOF MS spectral fingerprints based on the ribosomal protein database, 2) MALDI-TOF MS spectrum is converted to amino acid sequences based on the ribosomal protein database, 3) the conversion is verified by an independently constructed database based on the results obtained by sequence analysis of the $S 10$ spc-alpha operon.

\section{Conclusions}

This study scientifically revealed the function of $\mathrm{APEO}_{\mathrm{n}}$ and that harmless $\mathrm{APEO}_{\mathrm{n}}$ is biotransformed to metabolic toxicants with diverse chemical structures whose degree is dependent on microbial diversity influenced by environmental conditions. This study advances the idea that external inputs, such as minerals in the environment, modulate a specific response of the microbe community by regulating the population size of a specific microbe involved in controlling the biodegradation of chemicals. Such microbial plasticity in the environment can weaken or strengthen communication between microbes and external inputs under the stress of the chemicals. The LCIA of the chemicals would play an important role in fully understanding the environmental issues arising from the chemicals. 